\title{
La biografia de Philipp Bozzini (1773-1809) un idealista de la endoscopia
}

\author{
Verger-Kuhnke AB, Reuter MA, Beccaria ML. \\ Reuter Klink, Servicio de Urología del Hospital Karl-Olga, Hospital escuela de la Universidad de Ulm. \\ Museo de la endoscopía Max Nitze, Stuttgart.
}

Actas Urol Esp. 2007;31(5):437-444

\section{RESUMEN}

LA BIOGRAFIA DE PHILIPP BOZZINI (1773-1809) UN IDEALISTA DE LA ENDOSCOPIA

Philipp Bozzini nació el 25 de mayo de 1773 en Mainz, Alemania. El 12 de junio de 1797 obtiene el título de doctor en medicina. A partir de 1804 se dedica practicamente de completo al desarrollo de su instrumento; éste posee la forma aproximada de una vasija metálica de $35 \mathrm{~cm}$ de altura, revestida en cuero. En su cara anterior tiene una abertura circular que se encuentra dividida verticalmente por un tabique. En la mitad izquierda se encuentra la fuente lumínica (una vela de cera) y por detrás se encuentra un espejo, que proyecta la luz producida hacia el interior de la cavidad corporal a explorar. En la otra mitad, el observador recibe la luz reflejada y la imagen del órgano explorado. En la cara posterior se adaptan según la cavidad diversos espéculos, éstos permiten inspeccionar oído, uretra, recto, vejiga femenina, cuello uterino, boca, fosas nasales o heridas. Philipp Bozzini, logró con los modestos medios disponibles a principios del siglo XIX, demostrar al mundo médico el camino de la endoscopia. El estaba con su instrumento e ideas, tres cuartos de siglo adelantado a las posibilidades técnicas y científicas del momento. Los historiadores están de acuerdo, en que este instrumento, con luz artificial, diversos espejos y espéculos fue el comienzo de una numerosa familia de endoscopios ${ }^{9}$.

Palabras clave: Philipp Bozzini. Conductor luminico. Endoscopia.

\section{ABSTRACT}

BIOGRAPHY OF PHILLIP BOZZINI (1773-1809) AN IDEALIST OF THE ENDOSCOPY

Philipp Bozzini was born the 25 of May of 1773 in Mainz, Germany. The 12 of June of 1797 obtain the title of medicine doctor. From 1804 it is practically dedicated of complete to the development of its instrument, this have the approximated form of one metallic vase of $35 \mathrm{~cm}$ height, had in leather. In its previous face it has a circular opening that is divided vertically by a partition. In left half is the luminance source (a wax candle) and behind is a mirror, that it projects the light produced towards the interior of the corporal cavity to explore. By other half, the observer receives the reflected light and the image of the explored organ. In the later face they adapt according to the cavity diverse specula's, this allow to inspect ear, urethra, feminine bladder, rectum, uterine neck, nasal or wounds. Philipp Bozzini, profit with modest means available at the beginning of XIX century , to demonstrate to the medical world the way of endoscopes. It was with its instrument and ideas, 3/4 of century advanced to the technical and scientific possibilities of the moment. The historians are in agreement, in which this instrument, with artificial light, diverse mirrors and specula's war the beginning of a numerous family of endoscopies ${ }^{9}$.

Keywords: Philipp Bozzini. Light conductor. Endoscopy.

$\mathrm{B}$ ozzini vivió hace 200 años, en una época donde recién comenzaba la revolución industrial. La medicina no estaba todavía dividida en las distintas especialidades y subespecialidades como las conocemos hoy en día.
El término "Urologia" aparece por primera vez en un texto publicado por Johann Junker hacia 1738 en Halle y derivaba del vocablo "uroscopia".

El término "endoscopia" fue creado recientemente en 1853 por el médico cirujano y urólogo francés Desomeaux. 
El instrumento creado por Philipp Bozzini, tenía la finalidad de ser utilizado con diversos propósitos, como la exploración del oído, uretra, vejiga femenina, vagina, cuello uterino, cavidad bucal, fosas nasales, conducto rectal, heridas y trayectoria de proyectiles.

\section{BIOGRAFÍA}

Philipp Bozzini nació el 25 de mayo de 1773 en Mainz, Alemania. Su padre Nicolaus Maria Bozzini de Bozza provenía de una familia italiana acomodada que tuvo que huir de Italia hacia 1760 como resultado de un duelo. En Mainz, se dedica a los negocios y se casa con Anna Maria Florentin de Cravatte, oriunda de la ciudad de Frankfurt.

Bozzini comenzó sus estudios de medicina en Mainz y hacia 1794 se traslado a Jena para completarlos. El 12 de junio de 1797 obtiene el título de doctor en medicina, lo que lo habilita para establecerse en Mainz como médico. Él realiza poco después, con la finalidad de adquirir experiencia profesional, distintos viajes a Francia y Holanda $^{2,12}$.

En 1798 contrae matrimonio con Margarete Reck y tienen 3 hijos.

Durante la segunda guerra de coalición contra Francia, prestó sus servicios al ejército imperial y estuvo a cargo de un hospital de campaña con 120 camas en Mainz. Sus méritos extraordinarios durante esta época fueron conocidos por el Archiduque Karl de Austria (1771-1847) quien en un futuro protegería el invento de Bozzini. Éste pensaba que sería posible incorporar el instrumento en los hospitales militares austriacos; para ello era necesario enviar un aparato a Viena y efectuar además un peritaje por las autoridades sanitarias. Una comisión investigadora sometió el instrumento a diversas pruebas, comenzando por la exploración en cadáveres de la vejiga, recto, vagina, y cavidad peritoneal a través de pequeñas laparotomías. La junta propone algunas modificaciones con la intención de mejorar las prestaciones del conductor lumínico. Una vez llevadas a cabo quedan satisfechos con el funcionamiento del instrumento en pacientes (sólo las exploraciones de la cavidad peritoneal quedaron sin autorizar) sobre todo también por lo indoloro del método.
Debido a intrigas en las esferas superiores del gobierno, se decide efectuar un segundo peritaje, esta vez es la facultad de medicina en Viena, que la lleva a cabo y en parte bajo la negativa influencia de la iglesia ya que el informe se torna desfavorable para Bozzini, y concluye que un instrumento semejante no debería utilizarse ${ }^{7}$.

Con el tratado de paz de Luneville en 1801 entre Napoleón y el Kaiser Franz termina la segunda guerra de coalición, quedando la orilla izquierda del Rin en manos francesas. El nuevo gobierno en Mainz le otorga al joven Bozzini el permiso para ejercer la profesión, pero él se niega a recibir la ciudadanía francesa y decide por ello radicarse en Frankfurt ${ }^{9}$ (Fig. 1).

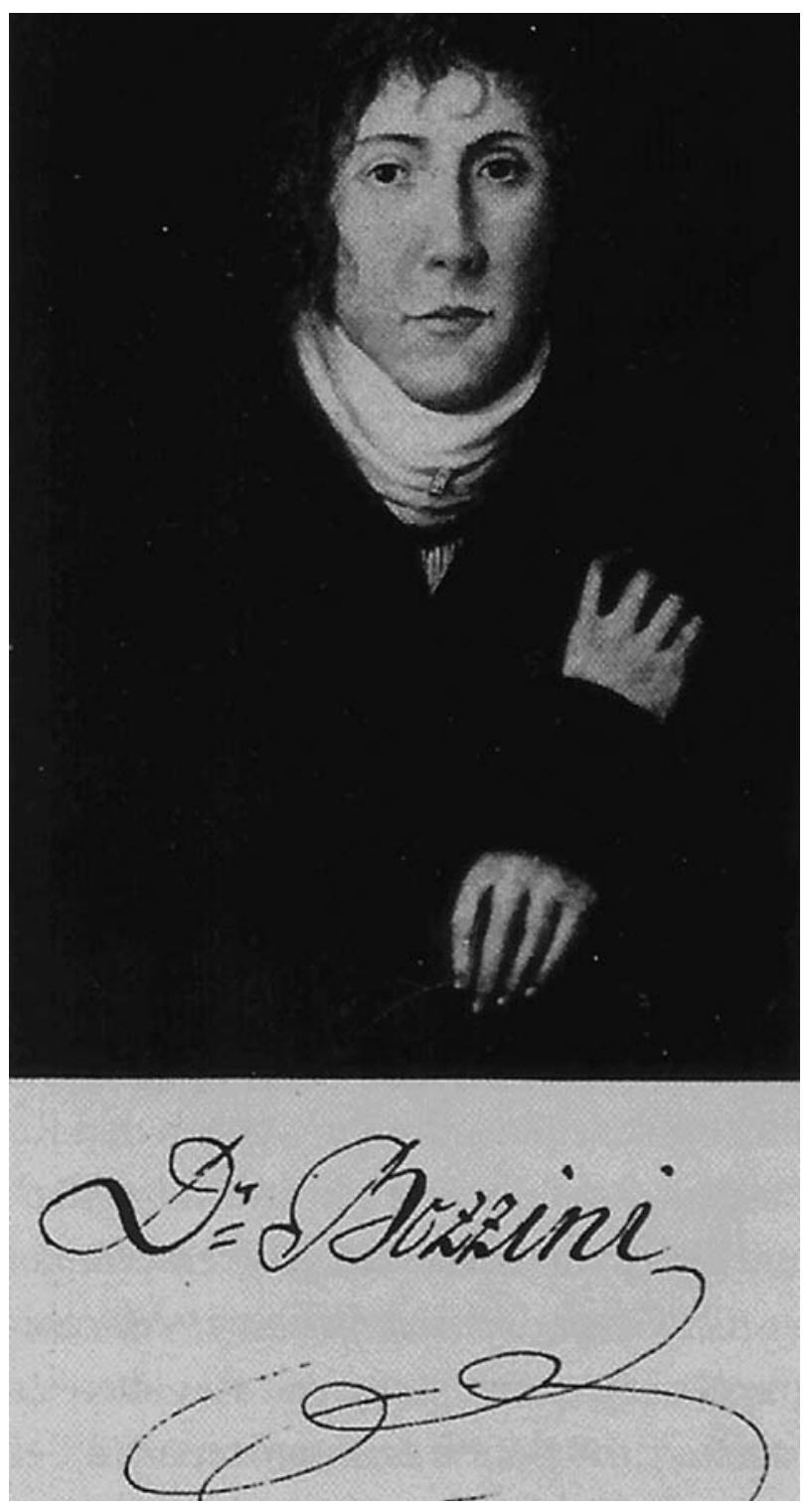

FIGURA 1. Retrato de Philipp Bozzini. 


\section{ACTIVIDADES DE BOZZINI EN FRANKFURT}

Sus conocimientos en matemáticas, filosofía y química eran admirables. Estudios aeronáuticos y dibujos de un aparato volador, lamentablemente se perdieron.

Su talento excepcional como artista y dibujante queda demostrado en su monografía sobre el “conductor lumínico", donde se aprecia un autorretrato y acuarelas sobre el instrumento ${ }^{6}$.

Como muchos idealistas, Bozzini era inexperto en asuntos comerciales, pero se dedicaba con entusiasmo a sus actividades científicas. A partir de 1804 se dedica prácticamente por completo al desarrollo de su instrumento para endoscopia. Para ganarse la vida se dedica con esmero a la obstetricia.

El 30 de Mayo de 1808 se le otorga el título de "Physicus extraordinario" a petición de uno de sus pacientes, Karl von Dalberg, personalidad de gran influencia en la región.

Bozzini era uno de los 4 médicos de la ciudad de Frankfurt que debía además atender las comarcas campesinas de los alrededores al mismo tiempo que era médico de "pestes".

Estas diversas tareas no eran solamente tediosas sino también peligrosas, su predecesor en el puesto el Dr. Zeitmann había muerto durante uno de los brotes epidémicos de tifus en la región.

Bozzini contrajo la misma enfermedad hacia mediados de marzo de 1809 después de haber tratado con éxito a 42 pacientes con tifus. $\mathrm{Su}$ amigo y colega Feyerlein relata posteriormente, con qué dedicación se ocupaba de sus pacientes, sin considerar el riesgo de contagio al que se sometía.

El 4 de abril de 1809 fallece Bozzini a los 36 años debido a esa infección. Él deja a su mujer en mala situación económica y ésta fallece 6 meses después. Sus 3 pequeños hijos fueron entregados a amigos.

Cuando se renovó el Domo de Frankfurt después de la guerra en 1954 se puso al descubierto la lápida recordatoria de Bozzini; en ella se puede leer todavía lo que le dedicó su amigo Feyerlein:

"A la devota alma de Philipp Bozzini, doctor en medicina, que fue el primero en explorar el interior de los órganos a través de su ingenioso pro- yector lumínico. Que supo combatir tenazmente la fiebre de los demás, con un gran sentido del deber, sucumbió en la noche del 4 al 5 de abril de 1809 en su $36^{\circ}$ año de vida. Su fiel amigo F.F. (Fig. 2).

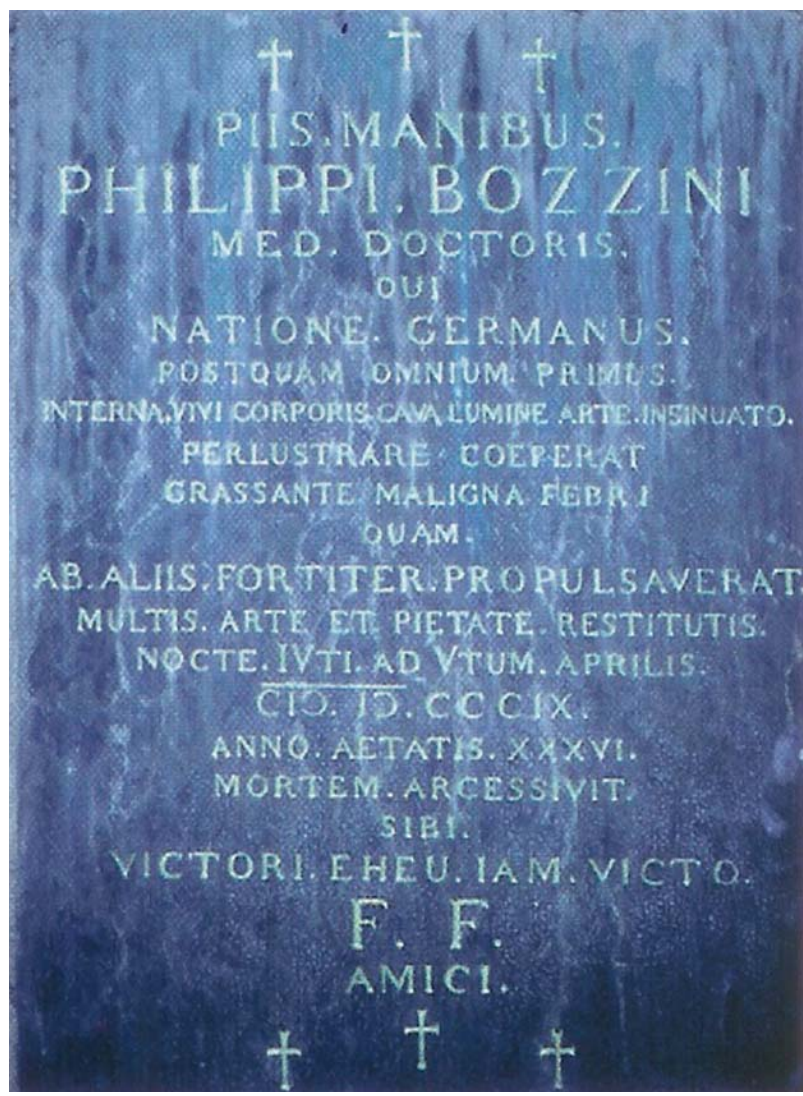

FIGURA 2. Lápida funeraria en el Domo de Frankfurt.

\section{EL “CONDUCTOR LUMÍNICO”}

(Der Lichtleiter)

La idea de observar el interior del cuerpo humano se remonta a la antigüedad. En un principio, se planteaba Bozzini la posibilidad de introducir en el cuerpo una sustancia fosforescente como la utilizada por ciertos indigenas en sus lámparas, la Vulgora laternaria y luego con la ayuda de un tubo o conducto poder reconocer algo.

Según el autor Ernst Rödiger en 1909, Bozzini había publicado ya en 1804 las características de su instrumento en un diario local de Frankfurt. En él describe que el aparato consta de una óptica, una fuente lumínica y una parte mecánica que se adapta a la abertura corporal que se desea explorar ${ }^{13}$. 
En esa época informa a diversos colegas amigos y obstetras, sobre el instrumento con la finalidad de que intenten la utilización clínica del mismo. Unos cuantos colegas se expresan de forma entusiasta sobre el nuevo instrumento, otros por el contrario lo hacen de forma negativa o acotando comentarios sarcásticos sobre el procedimiento.

En un paso siguiente publica las características del instrumento en un diario para gente adinerada, en el que describe la posibilidad de efectuar también operaciones a través del instrumento, en el interior del cuerpo y bajo visión, esto provoca una gran reacción en la comunidad.

La primera publicación científica de Bozzini es en el año 1806 con el título original: "Der Lichtleiter, eine Erfindung zur Anschauung innerer Theile und Krankheiten nebst der Abbildung"( El conductor lumínico, un invento para la observación de los órganos internos y sus enfermedades).

Un año después, en 1807 aparece en la ciudad de Weimar, la segunda publicación científica en forma de una monografia, con nuevos contenidos y figuras bajo el título original: "Der Lichtleiter oder die beschreibung einer einfachen Vorrichtung und ihrer Anwendung zur Erleuchtung innerer Höhlen und Zwischenräume des lebenden animalischen Körpers" (El conductor lumínico, su descripción y utilización para la iluminación de las cavidades internas del organismo animal vivo) (Fig. 3)

Se podría afirmar que el instrumento fabricado por Bozzini es el primer endoscopio construi$\mathrm{do}^{10,13}$.

Durante la presentación de su monografía, describe Bozzini de forma magistral, cómo se pueden explorar las diversas cavidades orgánicas del cuerpo animal vivo. Esto lo lleva de inmediato a enfrentarse con muchos médicos de la época que tienen una orientación natural-filosófica sobre el origen de las enfermedades. Bozzini por el contrario es de la idea, de efectuar estudios científicos exactos basados en la fisiología para explicar los fenómenos nosológicos.

El 10 de noviembre de 1806 presenta, ante la academia imperial y un gran auditorio, con éxito la exploración vaginal de una joven mujer con ayuda del conductor lumínico y la utilización de

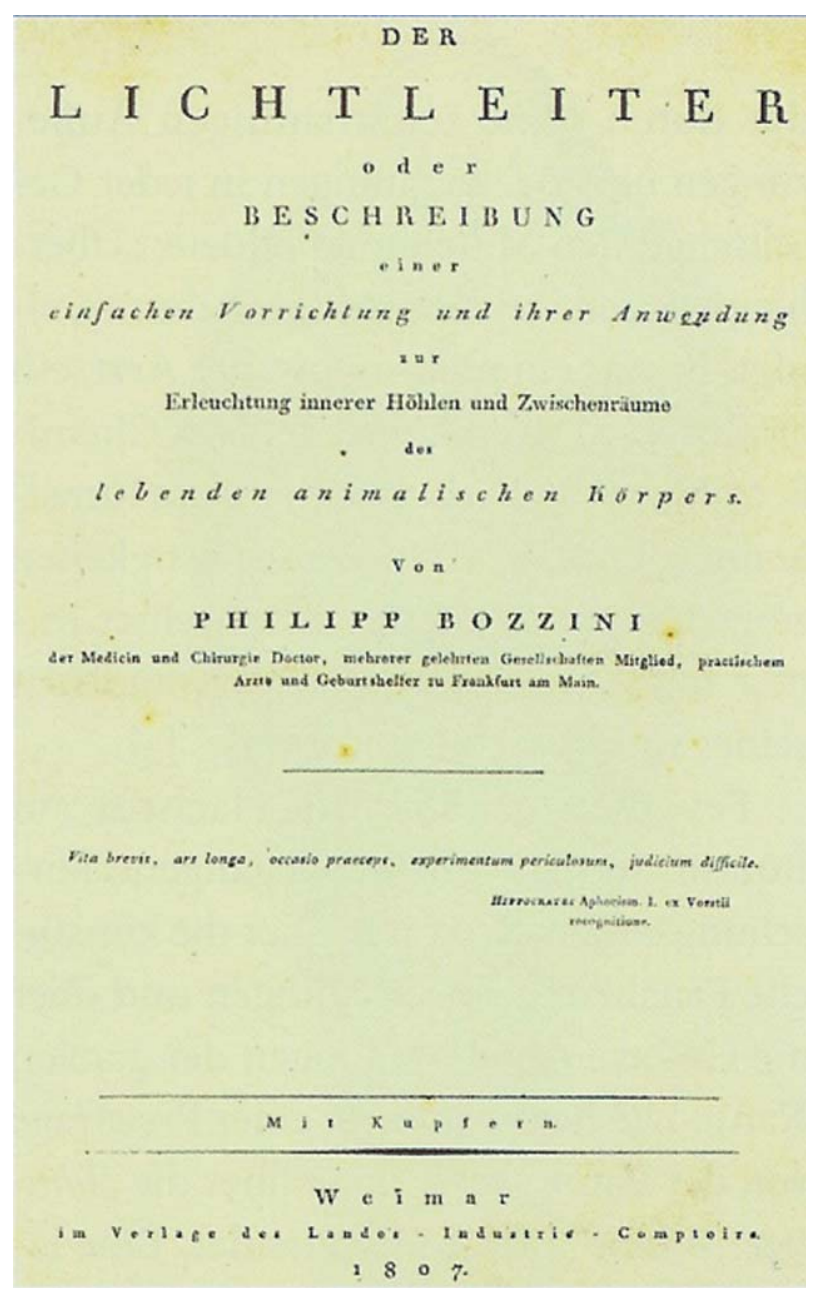

FIGURA 3. Publicación científica sobre el conductor luminico de 1807.

4 láminas metálicas (espéculos) adaptadas al mismo que permitían la apertura vaginal y la observación de la uretra.

\section{LAS CARACTERISTICAS TÉCNICAS DEL "CONDUCTOR LUMÍNICO"}

El instrumento consta de 2 partes fundamentales:

La óptica y un cuerpo contenedor de la fuente lumínica artificial (luz de vela).

Dispositivo mecánico en forma de tubo o conducto (disponible en diversas formas y tamaños) destinado a introducirse en las distintas cavidades corporales (espéculos) (Figs. 4 y 5)

$\mathrm{El}$ aparato posee la forma aproximada de una vasija metálica de $35 \mathrm{~cm}$ de altura, provista de cuatro caras, un pie y una pequeña chimenea en la parte superior, revestida de papel y cuero. En 


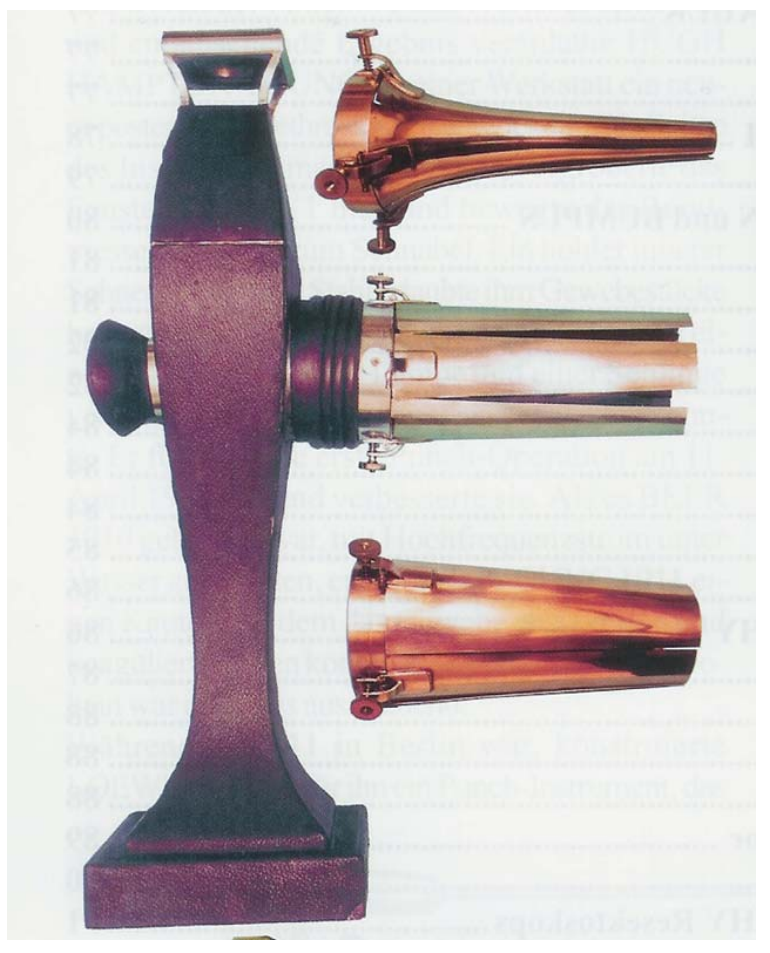

FIGURAS 4 y 5. Conductor luminico con los diversos espéculos.

su cara anterior tiene una abertura circular que se encuentra dividida verticalmente por un tabique. En una mitad se encuentra la fuente lumínica en forma de una vela de cera, a través de un resorte es posible que arda siempre a la misma altura (como en los faroles de las carrozas a caballo !!). Por detrás de la vela se encuentra un espejo, que refleja la luz producida hacia el interior de la cavidad observada. Por la otra mitad el observador recibe la imagen a través de una lente del objeto iluminado.

En la cara posterior y de acuerdo a la cavidad que se desea explorar por ej. el conducto auditivo externo, la uretra, la vejiga femenina o la trayectoria de proyectiles, se adaptan distintos espéculos. Estos constan de 4 hojas metálicas delgadas que, a través de un dispositivo con tuercas y tornillos permiten la apertura y dilatación del orificio a explorar. Para regiones corporales, que se mantienen ocultas a una visión directa, Bozzini construyo un artilugio a base de espejos cóncavos y planos que permitían así observar en un ángulo de $90^{\circ}$, por ejemplo el esófago, laringe, o las fosas nasales posteriores.

Para la exploración uretral creó un dispositivo que permitía a través de un catéter con una hen-

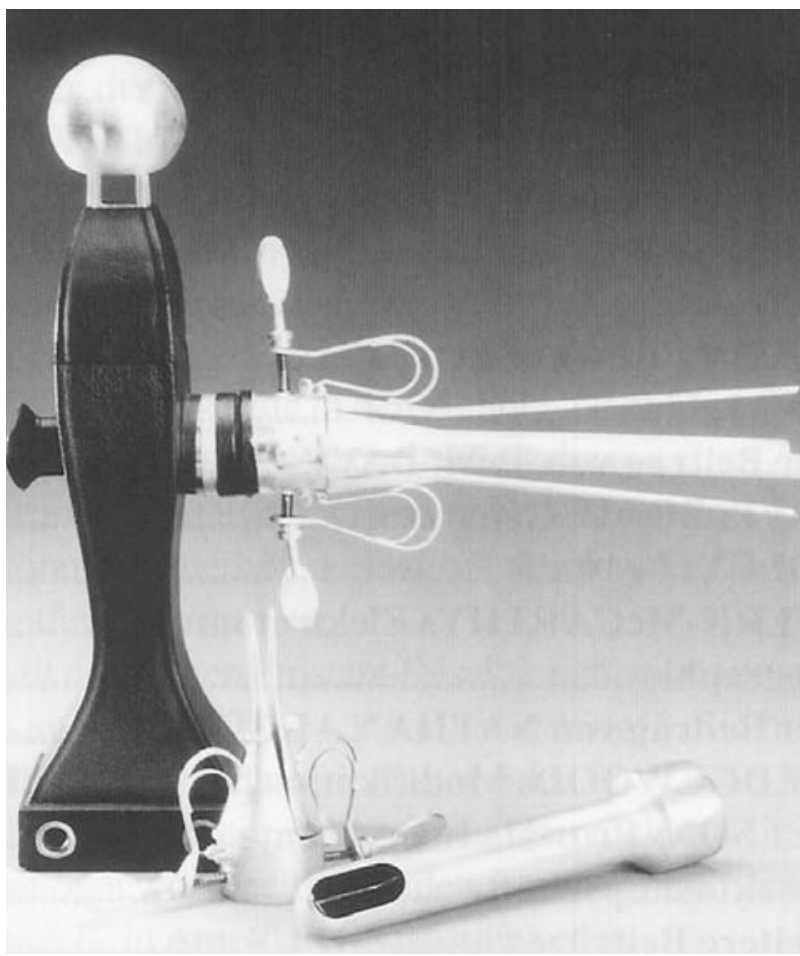

didura, efectuar hisopados, frotar una lesión o aplicar algún medicamento.

La parte superior del contenedor posee una chimenea que se puede equipar con una esponja humedecida con la intención de enfriar los gases producidos durante la combustión de la vela.

Los diversos espéculos se adaptan al contenedor en su cara posterior a través de un elemento elástico en forma de machete. Las dos caras laterales del instrumento carecen de dispositivos ${ }^{9,11}$ (Figs. 6 y 7).

\section{LA UTILIZACIÓN DEL “CONDUCTOR LUMÍNICO"}

Bozzini tenía ya una idea determinada de los órganos que se podían explorar, la cavidad bucal, conductos nasales y auditivos, la vagina, el cuello uterino, la uretra, la vejiga femenina y le recto. La vejiga masculina solo fue explorada por Bozzini a través de una cistostomía en cadáveres.

Bozzini pensó también desde un principio en operaciones endoscópicas, efectuadas a través de las aberturas naturales del organismo. El pensaba en retirar una de las cuatro hojas metálicas de los espéculos que mantenían el orificio corporal dilatado y a través de esa ventana introducir diversos instrumentos ${ }^{1}$. 


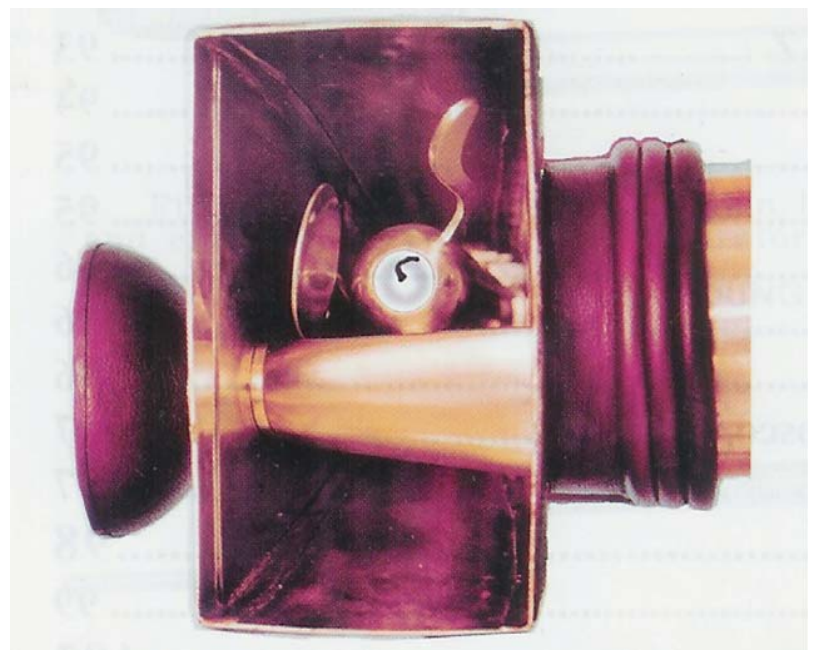

FIGURA 6. Instrumento visto de arriba.

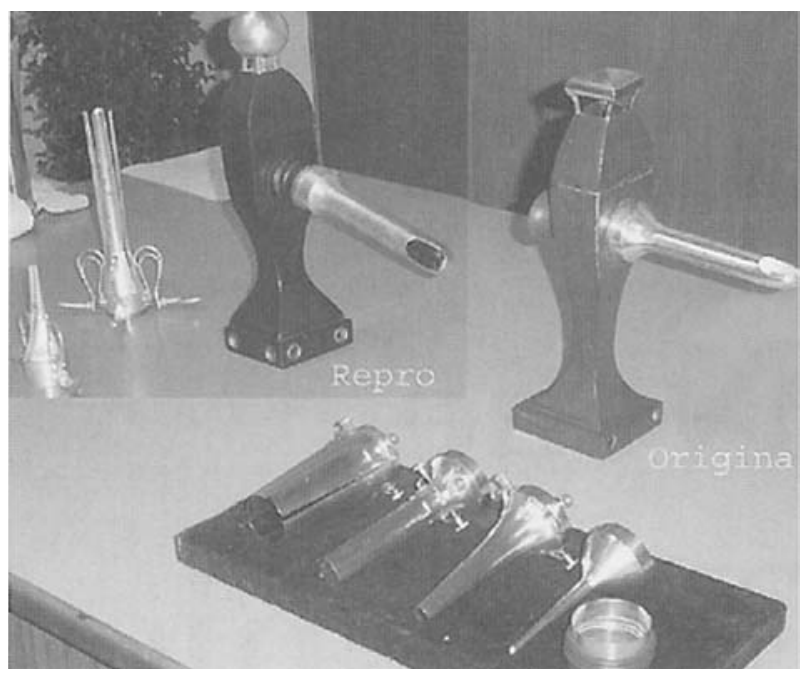

FIGURA 7. Conductor lumínico y espéculos.

El tenía también otras ideas más irreales o difíciles de llevar a la práctica, como por ej. la histerectomía transvaginal. El pretendía explorar heridas del abdomen y así diagnosticar las lesiones viscerales. También quería extraer cuerpos extraños o tratar lesiones orgánicas a través de suturas o ligadura de los vasos sanguíneos traumatizados. Pretendía explorar los cálculos vesicales tras la cistostomía y así poder determinar, forma, dimensión, número, etc. de los mismos.

Bozzini tenía claro, que a través de su instrumento se podía observar solamente un pequeño punto de la pared vesical femenina, aquí residía un problema más que ponía en evidencia las limi- taciones técnicas del momento. Con la introducción de prismas y lentes con aumento en un instrumento perfeccionado por Nitze ya se podrá efectuar una cistoscopia ${ }^{12}$ (Figs. 8 y 9).
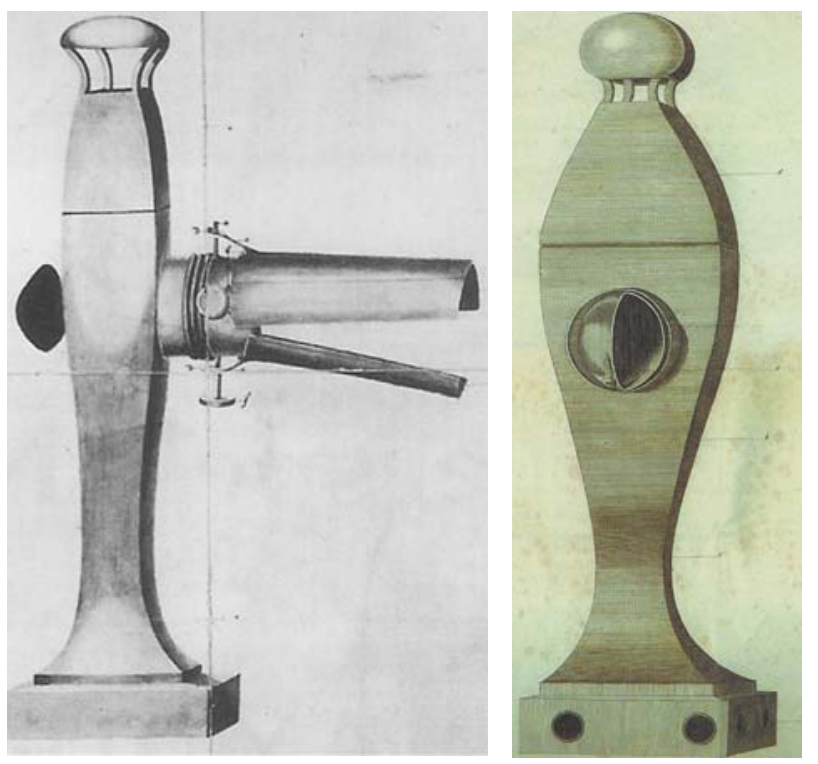

FIGURA 8 y 9. Conductor luminico de perfil y de frente.

\section{LOS COMIENZOS DE LA "ENDOSCOPIA"}

En junio de 1806 se efectuó la presentación del instrumento en la clínica privada de obstetricia del Prof. Friedrich von Froriep (1779-1847) en Halle ante docentes universitarios y médicos.

Se efectuó la exploración vaginal con el instrumento de Bozzini, y se pudo comprobar lo efectivo que era además de reconocer el potencial que tenía.

En contra de todas las críticas dirigidas al instrumento, le fue posible a Bozzini iniciar una discusión a nivel internacional, y acercar la idea de la endoscopia a un gran número de médicos.

En su libro sobre la historia de la endoscopia, describe Josef Grünfelds en 1879 los principios fundamentales del instrumento de Bozzini:

1. Luz artificial (vela)

2. Luz reflejada por un espejo hacia el objeto a explorar.

3. Observación por otro canal.

El punto débil de todos los instrumentos fabricados con el principio de Bozzini es la relativamente débil fuente lumínica ${ }^{5,6}$; además el calor y el humo generado por la vela ocasionaban moles- 
tias durante su uso y el peligro de sufrir quemaduras en la frente o en el muslo del paciente. Al inclinar el instrumento también se producían variaciones en la llama lo que ocasionaba dificultades visuales.

Posteriormente, con la finalidad de reducir todas estas molestias se utilizaron reflectores o fuentes lumínicas colocadas en la frente del observador.

En definitiva el instrumento de Philipp Bozzini es un buen ejemplo que demuestra que a veces las ideas se encuentran anticipadas a la época. La evolución desde que se obtiene una idea hasta la realización concreta es a veces un proceso dificil y largo.

A principios del siglo XIX las posibilidades técnicas de los constructores de instrumentos eran limitadas, además los materiales disponibles no eran los óptimos.

Sólo endoscopistas muy versados podían utilizar este instrumento en pacientes y obtener además algún provecho.

En 1853 presenta Antonin Jean Désormeaux (1815-1882) personaje perteneciente a una notable familia de médicos, un nuevo instrumento al cual él denomina por primera vez "endoscopio", pero basado en el mismo principio que el de Bozzini. El remplaza la débil luz de vela por una llama alimentada con una mezcla a base de petróleo, alcohol y terpentina, también incorpora un espejo con un agujero central e inclinado a $45^{\circ}$ sobre la llama como lo describiera Ségalas en sus instrumentos, que permitía así ver exactamente en la dirección donde se reflejaba el haz de luz ${ }^{3,4,14}$ (Figs. 10 y 11).

Con este nuevo instrumento le fue posible a Désormeaux efectuar exploraciones uretrales y vesicales, pudo diagnosticar cálculos y describir tamaño, cantidad, localización etc., también efectuó uretrotomías internas así como la coagulación de pólipos uretrales bajo visión.

Pero el instrumento era aún relativamente difícil de maniobrar lo que llevó al olvido del endoscopio por algunos años, hasta que Max Nitze retomara las ideas de sus antecesores e introdujera cambios e innovaciones para crear así el cistoscopio "moderno" 8 .

En los siguientes 150 años a la creación del "conductor lumínico" por Philipp Bozzini, las posibilidades técnicas fueron mejorando nota-

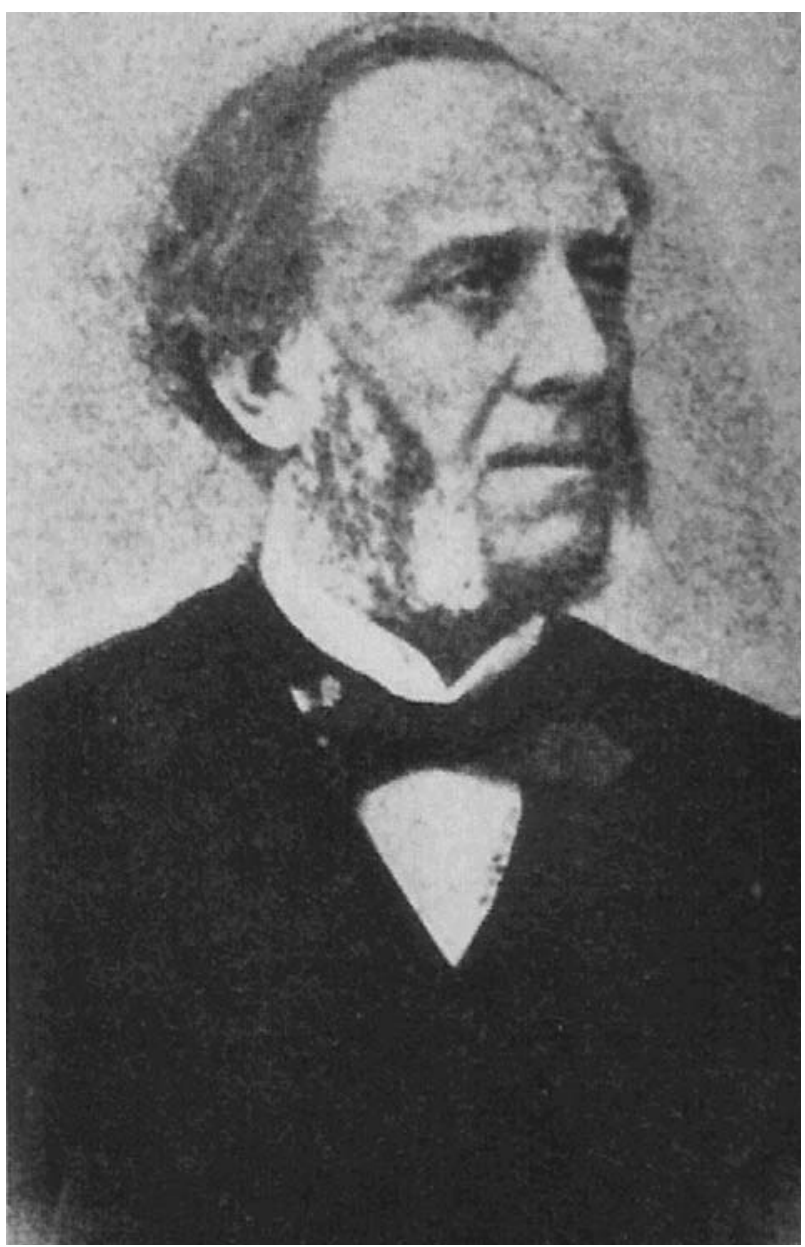

FIGURA 10. A. Désormeaux (1815-1882).

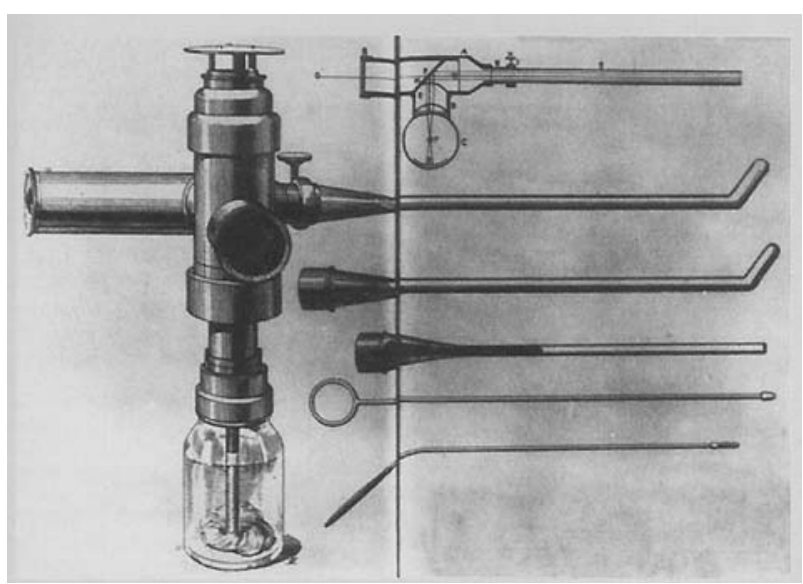

FIGURA 11. Primer "endoscopio" de Désermeaux 1853.

blemente, lo que facilitó la fabricación de instrumentos más adecuados y precisos que permitieron primero la exploración uretral, luego vesical y por último la observación endoscópica pielo-ureteral. 


\section{CONCLUSIÓN}

Philipp Bozzini, logró con los modestos medios disponibles a principios del siglo XIX, demostrar al mundo médico el camino de la endoscopia.

El estaba con su instrumento e ideas, a las posibilidades técnicas y científicas del momento tres cuartos de siglo adelantado.

Los historiadores están de acuerdo, en que este instrumento, con luz artificial de vela, diversos espejos y espéculos fue el comienzo de una numerosa familia de endoscopios ${ }^{9}$.

\section{REFERENCIAS}

1. Bozzini P (1806) Lichtleiter.J Pract Arzneykunde Wunderzneykunst 24:107.

2. Bozzini P (1807) Der Lichtleiter oder die Beschreibung einer einfachen Vorrichtung innerer Höhlen und Zwischenräume des lebenden animalischen Körpers. Verlag des landes Industrie Comptoir, Weimar.

3. Désormeaux MAS (1855) De l'endoscope, instrment propere a éclairer certaines cavités intérieures de l'économie. Comptes Rendus Hébdomadaires des Séances de l'Academie des Sciences 40:692.

4. Désormeaux MAS (1865) De l'endoscope et de ses applications au diagnostic et au traitement des affections de l'urethre et de la vaissie. Bailliere Paris.

5. Figdor PP (2001) Philipp Bozzini. Endo-Press, Tuttlingen Germany.
6. Grünfeld J (1879) Zur Geschichte der Endoskopie und der endoskopischen Apparate. Med Jahrb, Wien, Austria S 237-291.

7. Hauri D. Urologische Klinik, Universitästspital, Zürich, Swizerland. Ein Blick in der Blase-der Mensch dahinter. Der Urologe A 2005.44:401-407.

8. Mann G/1972) Altes Neues Philipp Bozzini (1773-1809). Medizinhist J7:201-203.

9. Reuter HJ, Reuter MA (1988) Bozzini und die Endoskopie des 19.Jahrhunderts. Thieme-Stuttgart-Germany.

10. Reuter MA (1998) Geschichte der Endoskopie. Krämer, Stuttgart, Germany.

11. Reuter MA (2004) Entwicklung der Urologie. Springer, Berlin, Heidelberg, New York.

12. Reuter MA (2006) Philipp Bozzini (1773-1809), Der endoskopische Idealist. Museum für Medizinische Endoskopie Max Nitze, Klinik für Urologie am KOK, Stuttgart, Germany. Der Urologe A 2006.45:1084-1091.

13. Roediger E (1990) Der Frankfurter Arzt Philipp Bozzini, der Erfinder des Lichtleiters (1773-1809). Alt Frankfurt 1: 4655 (reprint: 1972 Medizinhist J7:204-217.

14. Ségalas M (1827) Description of an instrument for inspecting the urethra and bladder. Lancet 7:603.

Correspondencia autor: Dr. AB Verger Kuhnke.

Reuter Klink, Servicio de Urología del Hospital Karl-Olga, Hospital Escuela de la Universidad de Ulm. Hack Str. 61 C.P. 70190 Stuttgart. Estado Federal de Baden-Württemberg-Germany.

E-mail autor: andresbverger@aol.com

Información artículo: Comunicación especial

Trabajo recibido: enero 2007

Trabajo aceptado: febrero 2007 